\title{
Personal styles and ways of coping in individuals who use wheelchairs
}

\author{
G Wheeler, R Krausher, C Cumming, V Jung, R Steadward and D Cumming \\ Rick Hansen Centre, and Departments of Educational Psychology, Obstetrics \& Gynaecology, and Medicine, \\ University of Alberta, Edmonton, Alberta, Canada
}

\begin{abstract}
To obtain information about the psychological needs of wheelchair users, 45 subjects participating in a university-based drop-in physical activity centre and outpatient rehabilitation program completed the Personal Styles Inventory and the Ways of Coping Inventory. Three groups of subjects were formed, differentiated by etiology for their disability. Results suggested that subjects with brain injury possessed the most limited coping resources. Subjects with multiple sclerosis were highest of the three groups in the frequency of utilization of emotional coping, they also revealed a fundamentally introverted and stability-based personality style. Subjects with spinal cord injuries utilized a predominantly Problem-solving means of coping; and they revealed a basic personality style characterized as extroverted and stable. The findings suggest that disease process (perhaps through common preexisting psychological characteristics, the nature of the onset and progression of the disease process, and/or tissue damage) may modify the ability to adapt and cope with the need to use a wheelchair. The nature of psychological support to be offered should reflect not only the use of a wheelchair but also the underlying personality and its modification by the nature of the disease process.
\end{abstract}

Keywords: wheelchair users; psychological profiles; spinal cord injury; brain injury; multiple sclerosis; rehabilitation

\section{Introduction}

Advances in medical technology and treatment have extended the life expectancy for many individuals who use wheelchairs and who formerly would have died as a result of their injury or from a secondary disease. Quality of life is now an important issue for the growing numbers of patients who may need to adapt to severe and chronic disabilities. For many persons, as their condition progresses or stabilizes, medical interventions are of limited benefit and management of the disability is likely to be carried out by the patients or their significant others. Therefore, the patient's ability to deal with their illness at a psychosocial level becomes a critical issue. Caregivers in the health system increasingly need an awareness of these issues to promote favorable adaption for their patients.

Psychological mechanisms such as ways of coping, locus of control and self-esteem may account, at least in part, for the type of adaptations ultimately made by persons with a disability. ${ }^{1,2}$ Researchers who have sought to describe the characteristics of individuals following a traumatic disability have tended to focus upon depression, social supports, self blame versus blaming others, self perceptions, internal or external

Correspondence: Dr DC Cumming, 1D1 Walter C Mackenzie Health Sciences Centre, University of Alberta, Edmonton, Alberta, Canada T6G 2R7 locus of control and other coping mechanisms in predicting the quality of adaption to chronic wheelchair use. ${ }^{3-8}$ There are also suggestions that individuals with a traumatic disability are more socially extroversive than those with a disability which resulted from a disease process ${ }^{9}{ }^{11}$ and have more effective adaptive coping resources in aiding in their adjustment to disability. ${ }^{12}$ Conversely, persons with a disability due to advanced degenerative central nervous system (CNS) disease (most notably, multiple sclerosis, MS) have been described as maladapted. ${ }^{13-19}$ Fatigue, depression, euphoria and uncontrollable emotional lability are some of the adverse symptoms which have been described in patients with MS. ${ }^{20,21}$ Marynov and Malkov ${ }^{21}$ reported that mental disorders are a significant component of the clinical picture of MS and were noted in $92.7 \%$ of their sample. They observed predominantly neurosis-like disorders, affective disorders and organic mental retardation. However, the findings from some studies of psychological characteristics and interpersonal functioning of patients with diagnosed MS have shown marked diversity. For example, Zeldow and Pavlou $^{22}$ (1988) developed a typology based on California Psychological Inventory ${ }^{23}$ factor scores and reported that a significant minority of those patients appear to be coping well.

In general, although the above studies emphasize 
the importance of psychological characteristics and psychosocial factors, there are discrepancies about the existence and nature of characteristics which might affect adaption to severe and chronic disability. While the review of the literature provides some information on psychological adaption, there is no consensus. It is reasonable to assume that prior to the injury or disease process varied personality and coping styles existed. The present exploratory research was part of a series of studies of individuals who use wheelchairs attending a university-based 'drop-in' physical activity and outpatient rehabilitation program. The aim was to determine if there was commonality of psychological characteristics within or among the groups; it was undertaken without prior assumptions of adaptation. We wondered whether the accumulation of factors such as the nature of the event which precipitated the disability (gradual and progressive versus instantaneous, trauma versus disease process, extent of brain injury, prognosis, or etiology) might influence the psychological status. The study was, therefore, exploratory and descriptive rather than hypothesis testing. It was designed to examine personality styles and psychosocial coping patterns of groups of subjects who use wheelchairs, differentiated by etiology for their using a wheelchair.

\section{Methods}

\section{Subjects and procedure}

Forty five consecutive new attendees at a physical rehabilitation centre were recruited for the study. Subjects were categorised as belonging to one of three groups of chronic wheelchair users (subjects with brain injuries [ $n=17]$, with multiple sclerosis $[n=9]$, or with spinal cord trauma, $[n=19])$. All were Caucasian adult men and women, living in an urban metropolitan area of approximately one million persons and currently attending a rehabilitation and activity centre for persons with a physical disability. The centre was non hospital based and was located in a university physical education faculty. The centre offered both individual and group rehabilitation; group programs were individualized according to ability and need and were not based on diagnosis.

Subjects gave informed consent for the study which had received the approval of an institutional ethical review body. They completed the Personal Styles Inventory (PSI-120) ${ }^{24}$ and the Ways of Coping Inventory (Revised). ${ }^{25}$ The questionnaires were administered individually by a single investigator prior to the rehabilitation activity session subsequent to the initial visit. While it is possible that individuals knew each other, communicated and discussed the questionnaires, it is unlikely that interpersonal contacts could influence responses since questionnaires were generally completed at the second visit to the facility prior to involvement in group activities. Self report demographic and health information was also gathered. Health ratings from hospital and medical records were not obtained, beyond requiring that the individuals attending the faculty have the consent of their medical advisor. A recent stressors scale was utilised in which life events over the preceding 12 months were given numerical values. The values allotted ranged from Loss through death to None. Scoring items were Loss through death $($ score $=1)$, Divorce $($ score $=2)$, birth of child $($ score $=3)$, marriage $($ score $=4)$, job loss $($ score $=5)$, change in job $($ score $=6)$, financial problems $($ score $=7)$, personal illness $($ score $=8)$, none $($ score $=9)$.

\section{Instruments}

The PSI-120 is a self-report inventory developed by Joseph Kunce and colleagues. ${ }^{24}$ The inventory can be completed in 15 to $20 \mathrm{~min}$ and provides information about basic (enduring) personality characteristics and more current behavioral tendencies and patterns. According to Kunce and colleagues, human personality co-varies along a two dimensional plane, characterized by introversion-extroversion and needfor-stability versus need-for-change. Basing the theory within the context of a circumplex model, they have described eight basic positions (8 major personality traits) that describe broad, general ways of behaving. Each trait is defined by personal styles in three different domains (ie, Emotional, Physical, Cognitive) which describe more specific ways of behaving.

The ways of Coping Inventory-Revised (WoC-R) is a 67-item inventory derived from the work of Lazarus and Folkman ${ }^{25}$ (1984) about the cognition of coping. The WoC-R inventory assesses an individual's personal coping strategies and takes approximately $10 \mathrm{~min}$ to complete. The inventory exists in two forms: it can be analyzed for the functions of coping as well as the types of coping. ${ }^{25}$ The form of the WoC-R inventory used in the present study revealed scores of preference for Problem-Solving (PS) versus an Emotional (E) focus in coping activities. An overall frequency measure of summed, or aggregate, coping strategies employed by the individual is also provided. Stressors in the areas of work, health, and family are measured by the inventory and ways of coping in each area are assessed. Two coping style scores for each of the three areas are obtained as well as the overall frequency measure of coping strategies employed.

\section{Data analysis}

Data analysis was undertaken with a multivariate analysis of variance for all groups and variables using SPSSX for the IBM. Further analysis was undertaken using a series of one way analyses of variance and calculation of $95 \%$ confidence intervals to differentiate significant differences in individual scores among groups. Categorical data was evaluated using Analysis of proportions. 


\section{Results}

\section{Demographic information}

The sample of subjects with brain trauma $(n=17)$ included persons with cerebrovascular accident, as well as those with closed head injuries (motor vehicle accident and falls from height). The sample of subjects with MS comprised 9 persons, the group with disability due to spinal cord trauma consisted of 19 subjects. On analysis of proportions, there were no significant differences among the groups in gender, marital status, education, income level, solitary living arrangements, previously seeking mental help, or recent hospitalization (all $P>0.05$ ). Analysis of variance did not suggest any difference in mean age or duration since onset of illness or injury. The mean ages in respectively the brain trauma group, the MS group and spinal cord trauma groups were $34.3 \pm 9.4$ (Standard Deviation), $44.7 \pm 9.1$, and $39.5 \pm 15.5$ years. Time since injury or onset of illness for the same groups were respectively $6.5 \pm 2.3,7.2 \pm 3.0$ and $5.5 \pm 2.5$ years. There were no significant differences among the groups in rating of current physical status (respectively $2.4 \pm 0.8,2.0 \pm 0.5$ and $1.7 \pm 0.6, P>>0.05)$. MS subjects scored higher on recent stressors than either of the other two groups $(7.0 \pm 3.5$ in MS subjects versus $3.6 \pm 3$ in brain trauma subjects and $4.3 \pm 3.6$ in cervical cord trauma individuals, $P<0.01$ ).

Table 1 Summary of differences in Personal Styles among three groups of individuals who use wheelchairs according to diagnosis

\begin{tabular}{|c|c|c|c|}
\hline Group and variable & Emotional & Cognitive & Physical \\
\hline Brain trauma & Undifferentiated & Undifferentiated & Stable \\
\hline Multiple sclerosis & Stable introvert & Introvert & Stable introvert \\
\hline Spinal cord trauma & Extrovert & Stable extrovert & Extrovert \\
\hline
\end{tabular}

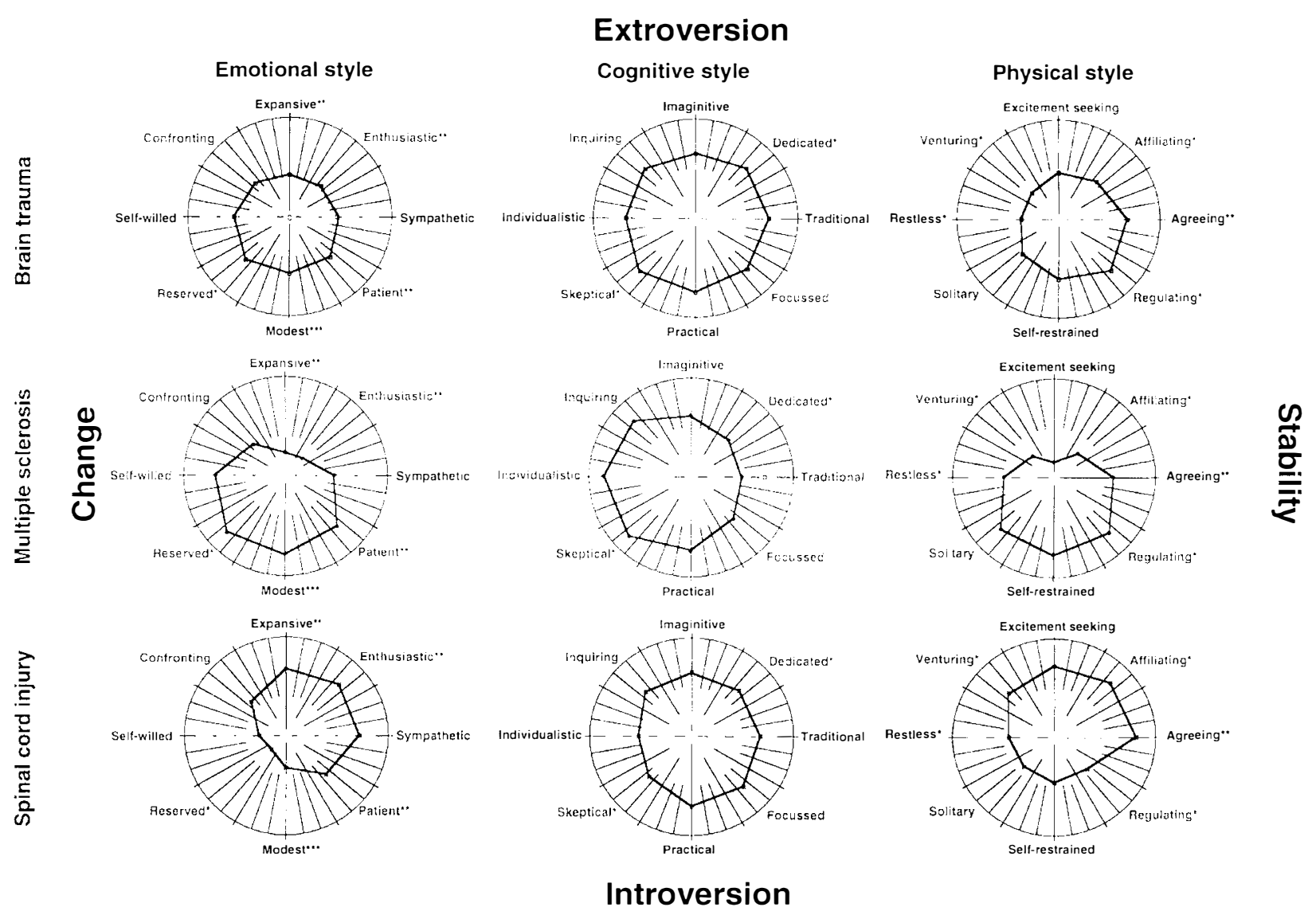

Figure 1 Polar display of personal coping styles mean scores in individuals using wheelchairs whose etiology was brain trauma, multiple sclerosis, or spinal cord trauma. The distance of points from the centre of polar display reflects scores on the scales i.e. points closer to the centre represent lower scores and as scale scores increase, the points are placed nearer the periphery of the circumplex diagram 
Personal styles inventory-numerical results

As summarized in Table 1, there were clear differences among the groups in personal styles. The octagonal polar plots of the results are shown in Figure 1.

Multivariate analysis of variance was significant on standard tests (Pillias $P<0.05$, Hotellings $P<0.001$, Wilks $P<0.01)$. The individual numerical results are shown in Table 2 together with the results of univariate analysis (Univariate $F$ tests). There were statistically significant differences (all $P<0.05$ ) among the groups on the Sympathetic, Expansive, Enthusiastic, Self-willed, Patient, Modest and Reserved Scales on the Emotional Style plot, on the Traditional, Dedicated, Individualistic and Skeptical Scales on the Cognitive Style Plot and on the Affiliating, Agreeing, Excitement-seeking, Solitary, Self-restrained, and Venturing Scales on the Physical Style Plot. Over $70 \%$ of subjects in each group conformed to the mean group profile.
Personal styles inventory - psychological interpretation of mean profiles

The mean profile of subjects with disability due to Brain Trauma had scores uniformly at the low end on the 8 Emotional scales and were in the terms of the PS120 'undifferentiated' in Emotional and Cognitive Styles (ie there were no sharply defined personality characteristics, possibly because their injury(ies) had substantially altered personality adjustment and functioning). In Physical Style, mean scores suggest that these individuals seek out familiar surroundings and social situations, acting in a cooperative manner in order to keep things going smoothly. They are not restless, or impatient with set schedules and timetables. They are also not comfortable with and avoid seeking out work that can be done alone, being neither autonomous nor solitary. Their Physical Style is thus stability oriented. Overall, the profiles of the Brain Trauma subjects suggest lack of a well defined style for relating to others or dealing with situational factors.

Table 2 Numerical scores of personal coping styles mean scores in individuals using wheelchairs whose etiology was brain trauma, multiple sclerosis, or spinal cord trauma. (Values in parentheses represent $95 \%$ confidence limits)

\begin{tabular}{|c|c|c|c|c|}
\hline \multirow[b]{2}{*}{ Scale } & \multicolumn{3}{|c|}{ Diagnostic groups } & \multirow{2}{*}{$\mathrm{P}^{-}$} \\
\hline & Brain trauma $(\mathrm{n}=17)$ & Multiple Sclerosis $(\mathrm{n}=9)$ & Spinal cord trauma $(\mathrm{n}=19)$ & \\
\hline \multicolumn{5}{|l|}{ Emotional style } \\
\hline Sympathetic & $46.1(44.4-47.8)$ & $48.1(36.7-59.5)$ & $71.7(64.0-78.9)^{\mathrm{a}}$ & $<0.01$ \\
\hline Enthusiastic & $43.7(39.6-48.1)$ & $24.1(9.9-38.3)$ & $73.3(67.8-78.8)^{b}$ & $<0.01$ \\
\hline Expansive & $42.2(36.4-50.0)$ & $23.2(10.2-36.2)$ & $67.5(62.0-73.0)^{b}$ & $<0.01$ \\
\hline Confronting & $47.6(39.3-55.9)$ & $43.6(32.2-55.0)$ & $48.1(42.5-53.7)$ & NS \\
\hline Self willed & $54.4(46.9-61.9)$ & $86.1(73.7-98.5)$ & $26.5(21.7-31.3)^{b}$ & $<0.01$ \\
\hline Reserved & $60.4(54.4-60.4)$ & $80.6(72.0-89.2)$ & $19.4(14.0-24.8)^{b}$ & $<0.01$ \\
\hline Modest & $57.4(51.5-63.3)$ & $79.2(70.0-89.2)$ & $32.8(26.9-38.7)^{b}$ & $<0.01$ \\
\hline Patient & $57.1(49.6-64.6)$ & $71.7(61.8-81.6)$ & $55.8(50.0-61.6)$ & $<0.05$ \\
\hline \multicolumn{5}{|l|}{ Physical style } \\
\hline Agreeing & $54.2(45.0-63.4)$ & $47.0(39.2-54.8)$ & $64.9(58.2-71.6)^{\mathrm{c}}$ & $<0.05$ \\
\hline Affiliating & $42.9(36.2-49.6)$ & $26.9(17.9-35.9)$ & $62.5(55.4-69.6)^{\mathrm{b}}$ & $<0.01$ \\
\hline Excitement-seeking & $37.3(31.0-43.6)$ & $12.1(0.0-25.2)$ & $57.7(50.0-65.4)^{b}$ & $<0.01$ \\
\hline Venturing & $29.7(24.0-35.4)$ & $23.6(8.2-39.0)$ & $50.3(43.2-57.4)^{\mathrm{a}}$ & $<0.01$ \\
\hline Restless & $29.6(23.6-35.6)$ & $39.0(25.8-52.2)$ & $36.1(33.6-40.6)$ & NS \\
\hline Solitary & $39.7(34.3-45.1)$ & $58.4(48.9-66.9)$ & $34.2(27.8-40.6)^{\mathrm{e}}$ & $<0.01$ \\
\hline Self-restrained & $48.2(42.0-54.4)$ & $62.1(67.5-57.7)$ & $36.0(30.6-41.4)^{b}$ & $<0.01$ \\
\hline Regulating & $59.0(51.2-66.8)$ & $62.1(55.3-68.9)$ & $54.4(48.6-60.2)$ & NS \\
\hline \multicolumn{5}{|l|}{ Cognitive style } \\
\hline Traditional & $57.5(51.6-63.5)$ & $39.7(27.0-52.4)$ & $53.7(49.2-58.2)^{\mathrm{c}}$ & $<0.05$ \\
\hline Dedicated & $56.3(51.5-62.1)$ & $41.8(32.4-51.2)$ & $52.5(46.1-58.9)^{\mathrm{d}}$ & $<0.05$ \\
\hline Imaginative & $51.8(45.5-58.1)$ & $48.9(37.3-60.5)$ & $50.9(44.3-57.5)$ & NS \\
\hline Inquiring & $55.3(48.0-62.6)$ & $62.8(54.2-71.4)$ & $50.2(43.6-50.8)$ & NS \\
\hline Individualistic & $54.0(47.9-60.1)$ & $68.3(59.6-77.0)$ & $41.6(34.3-47.3)^{c}$ & $<0.01$ \\
\hline Skeptical & $60.6(53.5-67.7)$ & $67.6(56.0-79.2)$ & $46.3(52.0-40.6)^{\mathrm{a}}$ & $<0.01$ \\
\hline Practical & $59.5(52.9-66.1)$ & $59.8(46.6-73.0)$ & $56.8(51.0-62.6)$ & NS \\
\hline Focused & $58.0(52.7-63.3)$ & $47.7(34.6-70.8)$ & $57.3(53.2-61.4)$ & NS \\
\hline
\end{tabular}

* $P$ values given in the final column are from individual univariate analyses of variance to examine whether there were significant differences among groups which could then be further examined using $95 \%$ Confidence intervals. The keys to significant results on final post-hoc testing using $95 \%$ Confidence intervals were as follows: ${ }^{a}=$ Spinal Cord Trauma Group significantly different from other two groups; ${ }^{b}=$ differences among all three groups; ${ }^{c}$ Spinal Cord Trauma Group significantly different from MS group; ${ }^{\mathrm{d}}=\mathrm{MS}$ group significantly different from Brain Trauma Group; ${ }^{\mathrm{e}}=\mathrm{MS}$ group significantly different from both other groups. In some cases the overall ANOVA suggested significant differences were present among the groups but Post-Hoc testing failed to find the differences 
Table 3 Mean $( \pm \mathrm{SD})$ values for Problem Solving and Emotional Coping Styles of the Ways of Coping Scale

\begin{tabular}{|c|c|c|c|}
\hline \multirow[b]{2}{*}{ Scale } & \multicolumn{3}{|c|}{ Diagnostic groups } \\
\hline & Brain trauma $(\mathrm{n}=17)$ & Multiple Sclerosis $(\mathrm{n}=9)$ & Spinal cord trauma $(\mathrm{n}=19)$ \\
\hline Problem solving & $37.4 \pm 15.0$ & $38.3 \pm 14.7$ & $42.7 \pm 16.0$ \\
\hline Emotional & $27.5 \pm 12.2$ & $30.0 \pm 7.1$ & $24.0 \pm 10.4$ \\
\hline
\end{tabular}

There were no significant differences among the groups on either measure

In Emotional Style mean profile, subjects with Multiple Sclerosis appeared modest, unassuming, shy, and self-conscious. They seek to avoid attracting attention to the self and are prone to holding back personal feelings to avoid offending others. They are also tender and softhearted, reacting emotionally to events occurring around them. They are characterized as stable introverts. In Cognitive Style, this group is individualistic, doubting, skeptical in outlook, tending to re-evaluate and re-examine existing ideas and beliefs; tending to require personally observable evidence; tending to engage only in goal-directed and useful projects and tasks. They do not tend to place importance upon the opinions of experts. They are also not prone to think in abstract, ideological or imaginative ways. Again, they would be characterized as introverts. In Physical Style, they are regulating, cautious, seeking to enhance security through order and control, guarding against unneccessary risks to the self by being extra cautious and well-prepared. They seek out familiar and friendly situations. They do not seek out many activities and are not game or party oriented. They are also not venturesome or daring and are neither bold nor impulsive. They avoid any risky or dangerous activities.

In Emotional Style, subjects with a disability due to Spinal Cord Trauma are enthusiastic, cheerful, lively, open, and optimistic. They see 'the bright side' of events - even of failures. They are expansive and selfconfident, and amenable towards public involvement and participation. They are confronting and spirited, spontaneously reacting quickly to events as they occur. They are not reserved or quiet, modest or unassuming, or shy and self-conscious. They do not seek to avoid attracting attention. They are not prone to holding back or being controlled in expression of personal feelings. They are characterized as Extrovert. In cognitive style, they are conventional and traditional in outlook, viewing events in conservative, accepting ways. They tend to place importance upon the opinions of experts. They are capable of thinking in more abstract, general and imaginative ways. These individuals are not self-doubting and skeptical in outlook nor are they prone to re-evaluate and reexamine existing ideas and beliefs. These features would be characterized as stable extrovert. In Physical Style, these individuals seek out group activities. They are good team players and members. They are also excitement-seeking, people-oriented, venturesome, daring, bold, and impulsive. They are not solitary, seeking out individual activities. They are also not comfortable alone. Furthermore, they are not introspecting or selfoccupied. Also, they are not regulating, cautious, and overly concerned over safety from harm. Their Physical Style is, therefore, characterized as Extrovert.

A majority of individuals in each group conformed to the mean profile. The reproducibility of the profile remains to be established by further research.

Ways of coping inventory-revised (WoC-R)

As shown in Table 3, there were no differences in emotional or problem solving coping styles (both $P<0.05)$.

\section{Discussion}

The present study is the first which has employed the PSI and WoC-R together in a population with disabilities. Our goals in the study were to see if there were significant differences among the groups and to describe the mean profiles of each group. It may be anticipated that several factors could confound the findings in the present study. The personal styles and ways of coping could be influenced by individuals' varying functional abilities, age at onset, support system, employment status, quality of life, affective status and preinjury life stability. Since the epidemiological data showed minimal differences among the groups, such factors probably had little influence. The varying functional abilities of the individuals and the possibility of brain damage may have a more significant influence on ways of coping and personal styles. In the present investigation, we found distinct coping and personality differences for different diagnostic categories among this small group of patients with chronic disabilities. There is sufficient homogeneity in the responses within the groups and clear differences among the groups that, despite the confounding variables, statistically significant differences emerged. While the characteristics may differ within a group, it is possible to provide a summary of the differences among groups. The sample sizes in the present study are relatively small; an enhanced study should be undertaken to further examine the present findings.

Subjects with disabilities following brain injury possessed few distinctive characteristics, a lack of clear orientation and the most limited coping resources. Such limitations imply that persons with 
disability due to brain injury need to depend on others for help in many situations, consonant with clinical research literature that relates brain dysfunction and impairment to defects in self directed activities. ${ }^{26}$ Confusion and inability to problem solve is implied.

In persons with MS the pattern of scoring on the PSI suggests these individuals are likely to be critical, questioning and skeptical about explanations given by health care providers. They also appear to be rather moody, private and withdrawn persons, who may be tense and somewhat uncomfortable in many social situations. Thus, although the persons in this study with MS seem to be 'hurting' and feel distresses, they might have difficulty reaching out to others. These characteristics suggest a brooding discontent, which is not a favorable state when facing problems, as they adapt to their disability. The characteristics for persons with MS in the present investigations are similar to those in other studies of the prevalence of psychosocial disturbances. ${ }^{13-21}$

The subjects with disabilities due to spinal cord injury revealed a basic personality style characterized as extroverted and stable. Such individuals might readily seek and accept the help of others. Despite this outgoing quality, the assessment instruments used in this study further discriminated a possible tendency for patients who use a wheelchair following spinal cord injury to have a cautious and traditional way of dealing with problems and stresses. They seemed concerned about control and may prefer to carefully reason and plan activities in advance. It might be anticipated that such individuals are also reluctant to take any chances or try out new or innovative ways of compensating for their disability. Thus, their options during rehabilitation may be limited by this somewhat conservative approach. These observations reflect general correspondence with the findings of coping resources in patients with spinal trauma reported by Frank and colleagues. ${ }^{2}$

In contrast to previous studies, all subjects in the present study were attending a single non-hospital based rehabilitation centre, although the results are also compatible with previous findings about psychological adaptation in similar diagnostic groups studied separately. ${ }_{-12}$ Particular personality traits of the individuals with a disability due to MS and to brain injury in this study contrast with those shown by the spinal cord injury group. Such factors probably have a strong influence on those individuals as they cope and adapt to their condition. Other factors, including personality characteristics may be more relevant for some patients. The Personal Styles Inventory used in combination in this study, revealed the diverse styles of coping which are likely to be the major factors to consider when treating disabled patients with stabilized or chronic conditions. As such, we have begun to focus on a psychological approach to the individual interacting with their disease process rather than their means of transport.

There is an interesting discrepancy in the findings since WoC-R scale found no significant difference among the groups while the PS-120 found clear differences among the groups in some but not all areas under assessment. Since the WoC-R is an accepted test which is generally considered reliable, some doubt may be cast on the PS-120. It is difficult to dismiss the PS-120 findings as invalid because they differ from a test which is generally considered reliable since the PS-120 is clearly measuring some factors which are different among the groups. The PS-120 is more complex and extensive and perhaps for that reason the more reliable. It is difficult to say that one test is reliable while the other is not. The underlying philosophy of the tests differs and they are examining different areas. It remains to be seen how the use of the investigations develops.

Our findings on the PS-120 have implications for treatment. Persons with spinal cord trauma who tend to restrict their activities and are apprehensive about trying out anything unfamiliar, may need encouragement to engage in some new ventures. Since these individuals are inclined to respond positively to social support they are also likely to benefit from encouragement by health care providers. In contrast, the painful ruminations of the patients with MS and difficulty sharing their disquieting thoughts and feelings with others can constitute an ineffective coping style. In view of their socially distancing behaviors and analytical/questioning attitude, a cognitive approach and developing a trusting relationship with them over time, together with possible treatment for depression might relieve some of the MS patients' marked distress. Conversely, a more openly supportive approach and a structured environment could be beneficial for subjects with brain injuries.

In the future, comparative studies with other groups of subjects who have CNS disorders might reveal similarities or differences in personality traits and etiologic factors. Psychological treatments which are found to be beneficial for any of the groups with central nervous system disorders could be used with greater discrimination. Longitudinal studies could determine whether certain subjects with disability due to spinal cord trauma go through stages of adaption and need to be managed differently over time, by initiating psychological investigation soon after a traumatic injury has occurred and looking at potential changes in coping mechanisms for different phases of the recovery process. Studies could also examine the role of quality of life and affective status in determining the individual's ability to cope with the need for wheelchair use.

\section{References}

1 Frank R, Elliott T, Corcoran J, Wonderlich S. Depression after spinal cord injury: Is it necessary? Clin Psychol Rev 1987; 7: 611 630.

2 Frank R, Umlauf R, Wonderlich S, Askanazi G. Differences in coping styles among persons with spinal cord injury: A cluster analytic approach. J Consult Clin Psychol 1991; 55: 727-731. 
3 Crewe N, Krause J. An eleven year follow-up of adjustment to spinal cord injury. Rehabil Psychol 1990; 35: 205-210.

4 Bordieri J, Kilbury R. Self-blame attributions for disability and perceived rehabilitation outcomes. Rehabil Couns Bull 1991; 34: 320-331.

5 Craig A, Hancock K, Dickson H, Martin J. Psychological consequences of spinal injury: A review of the literature. Austr $N$ Z J Psychiart 1990; 24: 418-425.

6 Sholomskas D, Steil J, Plummer J. The spinal cord injured revisited: The relationship between self-blame, other-blame and coping. J Appl Soc Psychol 1990; 20: 548-574.

7 Heinemann A, Bulka M, Smetak S. Attributions and disability acceptance following traumatic injury: A replication and extension. Rehabil Psychol 1988; 33: 195-206.

8 Van den Bout J, Van Son Schoones N, Schipper J, Groffen C. Atributional cognitions, coping behavior, and self-esteem in inpatients with severe spinal cord injuries. J Clin Psychol 1988; 44: $17-22$.

9 Malec J. Personality factors associated with severe traumatic disability. Rehabil Psychol 1985; 30: 165-172.

10 Rohe D, Basford J. Traumatic spinal cord injury, alcohol, and the Minnesota Multiphasic Personality Inventory. Rehabil Psychol 1989; 34: 25-32.

11 Frank R, Wonderlich S, Corcoran J, Umlauf R. Interpersonal response to spinal cord injury. J Soc Clin Psychol 1987; 4: 447 460.

12 Nieves C, Charter R, Aspinall M. Relationship between effective coping and perceived quality of life in spinal cord injured patients. Rehabil Nurs 1991; 16: 129-132.

13 Schwartz L, Devine P, Schechter C, Bender A. Impact of illness upon lifestyle. Loss, Grief and Care 1990; 4: 3-21.

14 Long D, Miller B. Suicidal tendency and multiple sclerosis. Hlth Soc Work 1991; 16: $104-109$.
15 Danto B. Emotional stress and multiple sclerosis. Loss, Grief and Care 1990; 4: 75-88.

16 Gorres H, Ziegler G, Friedrich H, Lucke G. Illness and Stress: Forms of psychosocial coping in multiple sclerosis. Zeitschr Psychosomat Med Psychoanal 1985; 34: 274-290.

17 LaRocca N. Psychosocial factors in multiple sclerosis and the role of stress. Conference of the New York Academy of Sciences: Multiple Sclerosis: Theory and practice (1983, New York, NY). Ann N Y Acad Sci 1984; 436: $435-442$.

18 Walsh P, Walsh A. Self-esteem and disease adaptation among multiple sclerosis patients. J Soc Psychol 1987; 127: 669-671.

19 Arnaud-Castiglione R, Garcia A, Mesana M, Scotto J. About psychiatric troubles in the course of multiple sclerosis. Med Psychol 1988; 20: $1503-1505$.

20 Schiffer R, Rudick R, Herndon R. Psychologic aspects of multiple sclerosis. N Y State J Med 1983; 83: $312-316$.

21 Marynov IS, Malkov GF. Neuropsychiatric disorders in multiple sclerosis. Zh Nevropatol Psikhiatr (USSR) 1986; 86: 176-182.

22 Zeldow PB, Pavlou M. Physical disability, life stress, and psychological adjustment in multiple sclerosis. $J$ Nerv Ment Dis 1984; 172: $80-84$.

23 Gough H. Manual for the California Psychological Inventory. Palo Alto: Consulting Psychologists Press, Inc 1990.

24 Kunce JT, Cope CS, Newton RM. Personal Styles Inventory. J Couns Devel, 1991; 70: $334-341$.

25 Lazarus R, Folkman S. Stress, appraisal and coping. New York: Springer Publishing Co. 1984.

26 Noonan W, Evans R, Hendricks R. Using family and personal variates to predict patient adjustment in stroke rehabilitation. International J Rehabil Res 1988; 11: 57.60. 